\title{
APORTES TEÓRICOS PARA EL ANÁLISIS \\ DE LA COMPETITIVIDAD Y LA CADENA DE VALOR EN LAS MICRO Y PEQUEÑAS EMPRESAS
}

\author{
THEORETICAL CONTRIBUTIONS TO THE ANALYSIS OF \\ COMPETITIVENESS AND THE VALUE CHAIN IN \\ THE MICRO AND SMALL ENTERPRISES.
}

Mg. Yezelia Cáceres Cabana

Docente

Universidad Católica San Pablo / Arequipa - Perú

Escuela de Administración de Negocios ycaceres@ucsp.edu.pe MBA. María Castillo Ureta

Docente principal

Universidad Católica San Pablo / Arequipa - Perú

Escuela de Administración de Negocios mcastillo@ucsp.edu.pe

Lic. Gabriela Carpio Anconeira

Universidad Católica San Pablo / Arequipa - Perú

Escuela de Administración de Negocios gabriela.carpio@ucsp.edu.pe

\section{Resumen}

El presente artículo presenta una revisión de los aportes teóricos que explican los elementos estratégicos generadores de competitividad y de valor en las micros y pequeñas empresas las mismas que son fuente de empleo y constituyen un elemento de soporte económico para la mayoría de peruanos. Se discuten enfoques sobre la competitividad empresarial, sus determinantes y metodologías de análisis.

Palabras clave: competitividad, cadena de valor, MIPYMES. 


\begin{abstract}
The present article presents a review of the theoretical contributions to explain the strategic elements that generate value and competitiveness of the micro and small business that are a source of employment and constitute an element of economic support for the majority of Peruvians. Approaches on business competitiveness, its determinants and analysis methodologies.
\end{abstract}

Keywords: competitiveness, value chain, MIPYMES.

\section{Introducción}

La economía peruana ha venido experimentando un crecimiento sostenido durante los últimos años y las MIPYMES sin duda han jugado un rol importante en este suceso ya que como menciona Okpara y Wynn (citado en Avolio et al., 2011) «son los pequeños negocios considerados la fuerza impulsora del crecimiento económico, la generación de empleo y la reducción de la pobreza en los países en desarrollo».

Lázaro (2010) define a las MYPES como:

Aquellas unidades económicas, constituidas por una o más personas naturales o jurídicas, que poseen intereses comunes, con el propósito de desarrollar actividades de extracción, producción, comercialización de bienes o prestación de servicios, enmarcadas bajo cualquier forma de gestión empresarial o societaria, desarrollándose dentro de un escenario jurídico especial.

La legislación peruana en la Ley $\mathrm{N}^{\circ}$ 28015, denominada "Ley de promoción y formalización de la micro y pequeña empresa”, artículo 2, define a la MYPE como:
La unidad económica constituida por una persona natural o jurídica, bajo cualquier forma de organización o gestión empresarial contemplada en la legislación vigente, que tiene como objeto desarrollar actividades de extracción, transformación, producción, comercialización de bienes o prestación de servicios.

La principal característica que diferencia la microempresa de la pequeña empresa es el valor de sus ventas, ya que el primer estrato empresarial es aquel que tiene ventas de hasta 150 UIT y el segundo, aquel que tiene ventas de más de 150 UIT y hasta 1700 UIT como consta en la Ley $\mathrm{N}^{\circ} 30056$.

En el Perú las MIPYMES constituyen el 99.4\% del total de empresas de las cuales el 94.9\% representa a la microempresa y el $4.5 \%$ a la pequeña empresa. Ambas, a su vez, aportan al PBI el $4 \mathbf{2 . 1} \%$ de su producción y emplean alrededor del 59\% de la PEA ocupada (PRODUCE, 2015). Para el año 2016 generaron el 88\% del empleo en el sector privado (PRODUCE, 2016).

No obstante, estas empresas tienen serios problemas para permanecer y desarrollarse en un mercado cada vez más competitivo, tenemos así que el 6o\% de ellas muere a 
los dos primeros años de fundada. La motivación y el carácter emprendedor, características que generalmente poseen los empresarios, no son suficientes para tener una empresa sostenible en el tiempo, sino que será necesario analizar el uso y gestión de otros recursos y capacidades que son determinantes en la competitividad de las mismas (COFIDE, 2014).

Investigaciones como las de Estrada, et al., 2009 señalan que «las pequeñas y medianas empresas altamente competitivas son aquellas que innovan en sus productos, procesos y gestión, que tienen un nivel tecnológico superior y poseen un plan estratégico»; por otro lado Flores y González (2008) mencionan que la competitividad está relacionada con los mercados, la tecnología, los sistemas administrativos, la calidad y los recursos humanos. Así mismo, Cabrera A. et al, (2011) concluyen que para hablar de competitividad empresarial es importante analizar factores como la innovación, el capital intelectual, la calidad, la tecnología, el conocimiento del mercado, la investigación y desarrollo, la asociatividad (cooperación entre empresas), las estrategias empresariales, la diferenciación, la productividad, los precios, la gestión financiera, la cultura organizacional y el servicio. A su vez, está la metodología del Mapa de Competitividad del Banco Interamericano de Desarrollo, (BID), en el que se considera que para el análisis de la competitividad empresarial se debe tener en cuenta la planeación estratégica, la producción y compras, el aseguramiento de la calidad, la comercialización, la contabilidad y finanzas, los recursos humanos, la gestión ambiental y los sistemas de información.
Así también, tenemos el modelo de competitividad sistémica, propuesto por la Comisión Económica para América Latina y el Caribe, (CEPAL), en donde se modela el desempeño competitivo de las empresas a nivel meta, macro, meso y micro, considerando el diálogo y la toma de decisiones en forma conjunta de cuatro dimensiones.

El desconocimiento de los factores por parte de los diferentes actores económicos y la falta de potencialización de los mismos son aspectos determinantes que inciden en la competitividad de las MIPYMES, y que pueden llevarlos a salir del mercado mucho antes de lo previsto, o en el mejor de los casos, los lleva a tener que subsistir, lo que ocasionará mayor desempleo, informalidad y una menor contribución al PBI del país.

Sobre las fronteras entre las micro y pequeñas empresas, según Herrera (2011), los criterios que se utilizan para analizarlas son de dos tipos: cuantitativos y cualitativos. En el primero de los casos se toman en cuentan aspectos como: número de trabajadores, capitalización, activo neto y bruto, inversión de capital, comprendida la inversión en maquinaria y equipo, valor agregado, ingreso bruto y neto, ingreso procedente de las exportaciones, además de sueldos y salarios pagados. En el segundo caso se toman en cuenta: un tipo de gestión personalizado; la capacidad técnica, y la integridad moral del propietario y su familia.

\section{Competitividad}

El concepto de competitividad ha evolucionado a partir de la teoría de la ventaja comparativa, que implica tener los mejores 
recursos hacia las ventajas competitivas y capacidad para producir bienes y servicios.

Porter (1990) y Krugman (1994) señalan que las que compiten son las naciones, no las empresas; por lo que a un país lo hacen competitivo las empresas competitivas que lo conforman; conformándose estas como la base de la competitividad.

Para Solleiroy Castañón (2005), la competitividad es un concepto que presenta un sinnúmero de definiciones, por lo que no es fácil encontraruna en la que todos estén deacuerdo. La competitividad puede ser estudiada desde diferentes enfoques y disciplinas, en parte porque su utilidad reside en identificar vías para fomentar empresas que contribuyan a elevar los niveles reales de bienestar.

Kao (2009) sugiere que los países deben tener esta capacidad de crear bienestar en un entorno en el que las empresas puedan competir, de manera que el nivel de prosperidad se pueda mejorary retroalimentar con los resultados del proceso, comparado con los de otras naciones de desarrollo económico similar.

La President's Commission on Industrial Competitiveness, en su estudio de los años 1980, señala que la competitividad debe ser entendida como "el grado en que una nación puede, bajo condiciones de mercado libre, producir bienes y servicios que satisfagan los requerimientos de los mercados internacionales y, simultáneamente, mantener o expandir los ingresos reales de sus ciudadanos». (Citado por Cabrera, et al., 2011)

Michael Porter, (citado por Mora, 2013) reconoce que la competitividad reside en la ventaja competitiva y esto significa que se crea, ya que es necesario gestionar las distintas capacidades, a las que se tenga alcance, para entregar productos y/o servicios que los clientes valoren.

Abdel y Romo (2004) refieren que la competitividad es la capacidad de crear un entorno que favorezca el crecimiento sostenido de la productividad, y que se refleje en niveles de vida más elevados para la población. Esto incorpora factores macro, meso y microeconómicos en un contexto de integración en la economía global.

Por su parte, Estrada et al., 2009 mencionan que la competitividad «es la capacidad para lograr una mejor posición en el mercado en relación con los demás competidores de su sector, obteniendo buenos resultados de un modo sostenible en el tiempo».

El concepto de competitividad involucra componentes estáticos y dinámicos, aunque la productividad de un país está claramente determinada por la habilidad de sostener sus niveles de ingreso, también es uno de los determinantes centrales de los rendimientos de la inversión, el cual es uno de los factores clave para explicar una economía en crecimiento, según World Economic Forum (citado por Saavedra, 2012).

Por lo mencionado podemos afirmar en concordancia con Flores (2008) que la competitividad es un término multidimensional que no posee una definición específica, debido a los diferentes niveles y factores cualitativos y cuantitativos que intervienen en su determinación, asimismo comprende la existencia de diversos enfoques de análisis e indicadores para su medición. No obstante, 
se observa que si algo tiene en común es que es un concepto universal utilizado como fórmula para el crecimiento económico de los países y como condición necesaria cuando se trata de ganar participación en los mercados internacionales (citado por Saavedra, 2013).

\section{Competitividad Empresarial}

Saavedra (2013) menciona entre las principales definiciones a Rubio y Aragón (2006) quienes dicen que esta es la capacidad de una empresa para, rivalizando con otras, alcanzar una posición competitiva favorable que permita la obtención de un desempeño superior a las empresas de la competencia.

Lall et al. (citado por Saavedra, 2012) explican que las compañías compiten para captar mercados y recursos, miden la competitividad según su participación relativa en el mercado o su rentabilidad y utilizan la estrategia de la competitividad para mejorar su desempeño.

\section{Solleiro y Castañón (2005) postulan que:}

La competitividad es la capacidad de una organización para mantener o incrementar su participación en el mercado basada en nuevas estrategias empresariales, en un sostenido crecimiento de la productividad, en la capacidad inter empresarial para participar en negociaciones con diferentes instituciones y otras compañías dentro de su ambiente, en un ambiente competitivo determinado por el sector y el mercado de los consumidores y en políticas introducidas por los gobiernos nacionales y alianzas económicas regionales.
Para Abdel y Romo (2004) el concepto de competitividad deriva de la ventaja competitiva que tiene una empresa a través de sus métodos de producción y de organización (reflejados en el precio y en la calidad del producto final) con relación a los de sus rivales en un mercado específico.

Otra definición que no ha sido mencionada es la de Mathews (2010) en un informe para el Ministerio de la Producción, donde señala que:
A nivel institucional - empresarial, se define la competitividad como la capaci- dad que tiene una organización, pública o privada, con o sin fines de lucro, de lograr y mantener ventajas que le permi- tan consolidar y mejorar su posición en el entorno socioeconómico en el que se desenvuelve. Estas ventajas están defini- das por sus recursos y su habilidad para obtener rendimientos mayores a los de sus competidores.

La teoría de los recursos y capacidades se centra en conocer en mayor medida los factores internos de las micro y pequeñas empresas. Este campo ha sido investigado por diversos autores que han desarrollado propuestas metodológicas para evidenciar de manera cuantitativa y cualitativa el nivel de recursos y capacidades con los que cuentan estas empresas del sector productivo manufacturero. Se tiene así el cuestionario elaborado y aplicado por investigadores de la Universidad Nacional del Centro de la Provincia de Buenos Aires en la investigación titulada "Una Propuesta Metodológica para la Determinación de Capacidades Estratégicas en PYMES industriales" (Saavedra, 2014). Siguiendo esta línea, Carpio (2017) utiliza 
esta metodología para el análisis del sector manufacturero metalmecánico en Arequipa - Perú, concluyendo que las capacidades estratégicas de la MYPES se centran en las operaciones y el servicio post venta.

A nivel latinoamericano y para las MIPYMES, se cuenta con herramientas propias para la región, siendo la más utilizada, en diferentes investigaciones, la propuesta del BID con el Mapa de Competitividad y la propuesta por la CEPAL con el modelo de competitividad sistémica, entre otros.

El Mapa de Competitividad, desarrollado por el BID, ve a la empresa como un sistema integrado por áreas que al conectarse entre sí mejoran el desempeño de esta. Las áreas son: planeación estratégica, producción y operaciones, (cadena de valor), aseguramiento de la calidad, comercialización, (merchandising), contabilidad y finanzas, talento humano, gestión ambiental y sistemas de información; incluyendo así aspectos internos, que al encontrarse bajo el control de la empresa, pueden ser indicadores de su competitividad.

El modelo de competitividad sistémica, propuesto por la CEPAL, plantea que la competitividad debe ser entendida como un sistema conformado por cuatro niveles que se relacionan entre sí y que modelan el desempeño competitivo a nivel meta, macro, meso y micro, considerando el diálogo y la toma de decisiones en forma conjunta (Cabrera et al., 2011).

El modelo de competitividad sistémica, propuesto por la CEPAL (2001), plantea que la competitividad debe ser entendida como un sistema conformado por cuatro niveles que se relacionan entre sí y que modelan el desempeño competitivo a nivel meta, macro, meso y micro, considerando el diálogo y la toma de decisiones en forma conjunta (Cabrera et al., 2011).

El nivel meta se ocupa de los factores sociales, culturales y las estrategias de gobierno para el fomento de las empresas. Por lo que tiene como premisa la integración social, en otras palabras, la formación de estructuras en la sociedad que eleven la capacidad de los diversos grupos de actores con el objetivo de articular sus intereses y satisfacer, entre todos, los requerimientos tecnológico-organizativos, sociales, ambientales y aquellos que plantea el mercado (Cabrera et al., 2011).

El nivel macro se sustenta en la estabilización del contexto macroeconómico, entre las que podemos encontrar la política fiscal, comercial, monetaria, cambiaria, presupuestaria y de competencia, que posibilitarán la asignación eficaz de recursos, asimismo exigirán eficacia de las empresas (Saavedra et. al., 2013).

El nivel meso es el nivel más significativo para la empresa ya que en este se ubican las instituciones y políticas que definen su entorno empresarial. En otras palabras se refiere a la formación e integración de estructuras en función de políticas de apoyo específico ya que en este espacio se encuentra el Estado, las empresas privadas y las instituciones intermedias articulándose y generando ventajas competitivas tanto organizativas como empresariales (Saavedra et. al., 2013; Cabrera et al., 2011). 
Y en el nivel micro, la competitividad se encuentra en los esfuerzos que la empresa realiza para elevar este indicador. La forma en cómo gestione los factores internos la misma será determinante para la creación de ventajas competitivas. Estos factores puede ser: la capacidad de gestión, estrategias empresariales, gestión de la innovación, mejores prácticas, interacción en redes tecnológicas, logística empresarial e interacción de proveedores, productores y usuarios (Cabrera et al., 2011).

En ese sentido, estos enfoques sostienen que la creación de ventajas competitivas es el producto de un patrón de interacción compleja y dinámica entre el Estado, las empresas, las instituciones intermedias y la capacidad organizativa de una sociedad. También vincula elementos pertenecientes a la economía industrial, a la teoría de la innovación, a la sociología industrial y gestión económica desarrollada por la ciencia política (Esser et al., 1994; Meyer-Stamer, 1996, citado en Saavedra y Milla, 2012).

A nivel académico, estas propuestas han sido ampliamente utilizadas en diversas investigaciones para analizar los factores que determinan la competitividad de las micro, pequeñas y medianas empresas. Así, para el caso de México, Saavedra (2012) sugiere utilizar criterios tanto internos como externos; para los primeros utiliza el Mapa de Competitividad del BID y para los segundos, el enfoque de competitividad sistémica de la CEPAL. En base a diversos estudios concluye que la mayoría de estas empresas, en un porcentaje del $80 \%$ son de baja competitividad, esto en "La competitividad en el nivel micro de la PYME en el Estado de Queretaro, 2012"; por otro lado, que la competitividad de una empresa está relacionada con el tamaño (micro o pequeña) y con el sector económico (comercio, servicio o industria), en "Determinación de la competitividad de la PYME en el nivel micro: El caso del Distritito Federal, México 2013"; y finalmente, que las carencias internas de la PYME, en este estudio el sistema de información, contribuirían a una competitividad global baja, en "La competitividad sistémica de la PYME del Distrito Federal, México 2015”.

En Colombia, Mora (2013), con el objetivo de identificar las áreas de competitividad que presentan falencias en las MIPYMES, pertenecientes a los sectores comercial y servicios, utilizó la clasificación dada por el Mapa de Competitividad del BID y en base a diversos estudios concluye que, los sectores analizados cuentan con un alto nivel de competitividad, mostrando un buen desempeño en las ocho dimensiones que componen el instrumento.

El Ministerio de la Producción para la Agenda de la Competitividad 2014-2018 (2014), explica que:

La competitividad de las empresas está determinada fundamentalmente por la productividad, la cual depende de la eficiencia en los procesos de producción y de la calidad de los productos. En ese sentido, la competitividad empresarial se manifiesta como producto de la capacidades tanto técnico-productivas como de gestión para lograr el máximo rendimiento de los recursos disponibles.

Por su parte, Ávalos (2008) refiere que la competitividad es «la capacidad que tiene una organización para operar y crecer 
rentablemente, es decir crear valor para sus propietarios, en un mercado donde operan competidores exitosos».

A medida quevan pasandolosaños, el mundo se vuelve más globalizado, la demanda del mercado y entorno se va modificando y con ello la competitividad es cada vez más dinámica. A partir de esto la competencia es un proceso en constante cambio en el que las empresas buscan, continuamente, satisfacer a sus clientes, de forma que superen a sus rivales y tengan una mayor participación en el mercado. La conducta competitiva está basada en la acción competitiva de las empresas, que a su vez se define como un movimiento directo, específico y directamente observable en el mercado, iniciado por una empresa para mejorar su posición (Usero y Fernández, 2005).

Se puede así postular como competitividad empresarial a «la capacidad de una empresa u organización de cualquier tipo para desarrollar y mantener unas ventajas competitivas que le permiten disfrutar y sostener una posición destacada en el entorno socio económico en que actúan» (Observatorio de competitividad y clusters, 2018).

La competitividad es también la búsqueda de una posición relativamente favorable en un mercado, con respecto a los rivales, la cual le permitirá permanecer y expandirse. La ventaja competitiva es aquella que cuenta con un valor agregado, el cual es apreciado y permite el crecimiento de la organización. En ese sentido podríamos decir que para lograrla es necesaria gestionar ciertas actividades (D’Alessio, 2013). Además, la ventaja competitiva es impulsada por las diferencias en la capacidad de transformar estos insumos en bienes y servicios para obtener la máxima utilidad (Kogut, 1985). Para diagnosticar la ventaja competitiva es necesario definir una cadena de valor para la empresa de manera que esta le permita competir en un sector industrial (Porter, 1987).

\section{Cadena de valor y Teoría de los recur- sos y capacidades}

De forma complementaria, se han ido desarrollando otras herramientas para identificar elementos de competitividad; tenemos así la teoría de la cadena de valor, desarrollada por Michael Porter (1987), quien la define como:

Un conjunto de actividades cuyo fin es diseñar, fabricar, comercializar, entregar y apoyar el producto, para entender el comportamiento de los costes, así como de las fuentes actuales y potenciales de diferenciación; así pues la cadena de valor está conformada por actividades mediante las cuales se crea valor para los clientes.

Por otro lado, la cadena de valor y la forma en que desarrollan las actividades individuales manifiestan su historial, su estrategia, su enfoque en el establecimiento de la estrategia y las economías en que se basan dichas actividades, es así que la cadena de valor contiene el valor total y consta de actividades relacionadas con la creación de valor de margen.

Los elementos de la cadena de valor de Porter se dividen en dos grandes grupos: las actividades primarias y las actividades de apoyo, estas últimas respaldan a las primeras (Porter, 2013). 
Las actividades primarias son aquellas que tienen que ver con el desarrollo del producto, lo que involucra actividades divididas en cinco categorías genéricas. Cada una puede dividirse en sub capacidades bien definidas que dependen de la industria y de la estrategia de la corporación, (Quintero y Sánchez, 2006 y Porter, 1987).

De acuerdo a Porter (1987), son actividades primarias:

- Logística de entrada o interna: actividades que se implementan para la correcta recepción, almacenamiento y distribución de los insumos que se adquieren para elaborar los productos y/o servicios.

- Operaciones: actividad que involucra el desarrollo del producto como tal, se inicia con la recepción de los insumos para luego pasar a la planificación, en el que se detalla cómo se llevará a cabo todo el proceso de fabricación y mantenimiento de los equipos con los que se trabaje.

- Logística de salida o externa: se inicia cuando el área de producción libera el producto y este es ingresado en inventario como producto terminado, para luego ser distribuido al cliente.

- Marketing y ventas: comprende actividades que implica conocer el mercado al que se orienta, desarrollar estrategias para relacionarse con el cliente y la implementación de herramientas de promoción.
- Servicios de post venta: estas actividades pretenden reforzar el valor de un producto; asimismo, nos permite conocer la satisfacción del cliente.

Y son actividades de apoyo a la creación de valor:

- Infraestructura de la empresa: abarca actividades de la administración general como la dirección, la planificación, financiación, relación con los inversionistas, contabilidad.

- Administración del capital humano: incluye actividades necesarias para reclutar, contratar, capacitar, desarrollary crear el sistema de remuneración al personal.

- Desarrollo tecnológico: Incluye actividades relacionadas con la innovación del productoy/o los procesos utilizados para su fabricación. Se refiere a los niveles de investigación y desarrollo de la tecnología que la empresa es capaz de emplear en función de la accesibilidad y su capacidad financiera.

- Adquisición o compras: Son las actividades para adquirir los insumos y materias primas necesarios para fabricar los productos de la empresa. Así como también, otros suministros y activos fijos como maquinaria, equipo de laboratorio, equipo de oficina y edificios.

Respecto al tema, esta teoría no solo se ha utilizado para encontrar puntos críticos en la empresa y proponer una posible solución, sino también se ha aplicado a las cadenas productivas y de comercialización; 
dando origen al concepto de Cadena de Valor Agregado que se define como la generación de un bien o de un servicio desde la materia prima, pasando por las fases de transformación hasta el producto final (Observatorio de competitividad y clusters, 2018).

De manera complementaria, a la gestión estratégica de la cadena de valor, está el enfoque de la Teoría de los recursos y capacidades de Barney (1991 y 2011) que busca analizar en el interior de las empresas para poder explicar por quéalgunas son competitivas y otras no, teniendo las mismas posibilidades ya que comparten el mismo mercado y tienen acceso a los mismos inputs y a idéntica tecnología (Fuentes, 2007). Hitty Hoskisson (1999) la conceptualizan como «un conjunto de recursos, capacidades y aptitudes centrales heterogéneos que pueden utilizarse para crear una posición exclusiva en el mercado».

La premisa principal de esta teoría es que hay dos supuestos fundamentales, uno la heterogeneidad de las empresas con relación a la dotación de recursos que poseen y el otro referido a la persistencia en la heterogeneidad de las dotaciones de recursos dada su movilidad imperfecta, Barney (1991); siendo esto consecuencia de las diferentes dotaciones de recursos y capacidades de los que dispone cada empresa, que constituyen, por tanto, la fuente de obtención de mayores rentas (Fuentes, 2007).

El concepto de recurso (Camisón, 2012; Estrada et al., 2008 y Saavedra et al., 2013) se refiere a todos los activos de los que dispone una empresa y que se pueden clasificar en:

- Tangibles, como físicos, financieros, humanos y organizacionales.
- Intangibles explícitos, como conocimiento tecnológico explícito, recursos comerciales en forma de derechos o de activos registrados con valor de mercado, conocimientos explícitos de las personas, entre otros.

- Intangibles implícitos de carácter tácito, reflejados en conocimientos implícitos en las personas, que se expresan en know-how o capital humano.

Por otro lado, el éxito competitivo se debe al conjunto de recursos y capacidades que esta posee y que la hacen diferente de los demás competidores del sector, explicando una proporción significativa de la variabilidad en los resultados empresariales.

Los recursos que tienen importancia estratégica, son aquellos que tienen como características ser valiosos, escasos, difíciles de imitar y relativamente insustituibles, que puedan ser apropiables por la empresa y que sean sostenibles en el tiempo, lo que a su vez permitirá que exploten las oportunidades y neutralicen las amenazas del entorno (Barney, 1991).

Estos recursos por sí mismos no son proveedores deventaja competitiva, ya que depende de la habilidad con que se utilicen y gestionen. Es así que las capacidades permiten el despliegue coordinado de recursos transformándolos en productos con valor agregado favoreciendo a aspectos como la productividad, la calidad, la eficiencia, entre otros (Estrada et al., 2008 y Saavedra et al., 2013).

La teoría de los recursos y capacidades de las empresas, considera que los recursos internos de carácter intangible son una 
fuente de ventaja competitiva tan o más importante que los factores que se derivan de la estructura del sector o de los factores del entorno genérico.

Por otro lado, es importante mencionar que las capacidades descansan sobre los recursos, ya que las primeras contribuyen en aumentar el stock de las segundas. Por lo que, cuando se logra una integración de recursos y una coordinación de capacidades es que se están generando ventajas competitivas (Estrada et al., 2008).

Además de generar ventajas competitivas estas capacidades, basadas en el conocimiento favorecen a las empresas que tienen intenciones de internacionalizarse (Gnizy et al., 2014).

En este sentido resultan interesantes las aplicaciones de esta teoría a pequeñas y medianas empresas, donde el conocimiento y la edad de las entidades pueden ser influyentes para explicar su emprendimiento y crecimiento, así como su éxito (Anderson y Eshima, 2013).

\section{Conclusiones}

1. En base a lo investigado se puede afirmar que, no obstante la variedad y profundidad de las diferentes definiciones de competitividad hay una constante en ellas y es que no se puede hablar de crecimiento, tanto de un país como de una empresa, sin referirnos a la competitividad.

2. Para el análisis de la competitividad empresarial en las MIPYMES es útil considerar la teoría de la cadena de valor, ya que esta evidencia los puntos críticos generadores de valor y las fuentes de ventajas competitivas; es importante considerar también, la teoría de los recursos y capacidades, ya que esta resalta la importancia del conocimiento y utilización de los recursos y las capacidades para la gestión estratégica.

3. Si bien el presente trabajo se centra en dos conceptos fundamentales para el crecimiento, desarrollo y sostenibilidad de las MIPYMES: competitividad y cadena de valor, no hay que dejar de lado la importancia de otros aspectos cualitativos como son el comportamiento organizacional, la gestión del talento y los aportes multidisciplinarios de las ciencias exactas en el éxito de las empresas.

\section{Referencias}

(2015). Anuario Estadístico Industrial Mipyme y Comercio Interno.

(2016). Anuario Estadístico Industrial Mipyme y Comercio Interno.

Avolio, B., Mesones, A. , \& Roca, E. (2011). Factores que limitan el crecimiento de las micro y pequeñas empresas en el Perú. Revista Strategia de CENTRUM de la PUCP.

CDI. (2015). Obtenido de http://www.cdi.org. pe/InformeGlobaldeCompetitividad/index. html

CEPAL. (2001). Elementos de competitividad sistémica de las pequeñas y medianas empresas del Itsmo Centroamericano . 
COFIDE. (2014). Memoria Anual.

Cohen, M. (2012). La situación de las PYMES en América Latina . Obtenido de http:// copal.org.ar/wp-content/uploads/2015/o6/ peal.pdf

D’Alessio, F. (2013). El proceso estratégico. Un enfoque de gerencia - Segunda Edición. México DF: Pearson Educación .

Economía, I. P. (2015). Índice de Competitividad Regional. Obtenido de http://www.ipe.org. pe/documentos/indice-de-competitividad-regional-incore-2015

Economía, I. P. (2016). Índice de Competitividad Regional . Obtenido de http://www.ipe. org.pe/documentos/indice-de-competitividad-regional-incore-2016

Fernández, R., \& Bautista, P. (2014). Metodología de la Investigación. D.F, México: Editorial McGraw Hill.

Forum, W. E. (2015). The Global Competitiveness Report 2015-2016. Obtenido de http://www. pemudah.gov.my/documents/10124/178798/ Global_Competitiveness_Report_2015_2016. pdf

Herrera, B. (s.f.). Análisis Estructural de las MIPEs y PYMEs. Revista Quipukamayoc de la Facultad de Ciencias Contables Volumen 18 No 35 pp 69 ? 89 (2011). Lima : UNMSM - Perú.

Mathews, J. (2010). Competitividad: El significado de la competitividad y oportunidades de internacionalización para las mypes. Ministerio de la Producción.
Montoya, A., Montoya, I. , \& Castellanos, O. (2010). Situación de la competitividad de la Pyme en Colombia: elementos actuales y reto. Obtenido de http://www.redalyc. org/articulo.oa?id=180315651012

Mora, E. (2013). Tesis de Maestría: Nivel de competitividad de las MIPYMES de Bogotá. Análisis por dimensiones estratégico. Bogotá - Colombia.

Moyano, J., Puig, F. , \& Bruque, S. (2008). Los determinantes de la competitividad en las cooperativas. Obtenido de http://www. redalyc.org/articulo.oa?id=17412302011

Paravié, D., Rohvein, C., Urrutia, S., Roark, G., \& Ottogalli. (2012). Diseño de un instrumento para evaluar el desempeño de las actividades que integran la cadena de valor de pymes metalmecánicas de Olavarría. Obtenido de https://dialnet.unirioja.es/

Producción, M. d. ( 2015). Las MIPYMES en cifras, 2014. Obtenido de http://www.produce.gob. pe/remype/data/mypezo14.pdf

Producción, M. d. (5 de julio de 2018). Las MIPYMES en cifras, 2016. Obtenido de http://ogeiee.produce.gob.pe/index. php/shortcode/oee-documentos-publicaciones/publicaciones-anuales/ item/758-las-mipyme-en-cifras-2016

ProInversion. (s.f.). Agencia de Promoción de la Inversión Privada ? Perú (2013). Ley $N^{\circ}$ 30056 . Obtenido de http://www.proinversion.gob.pe/RepositorioAPS/o/o/arc/ MOXI_LEY_30056/ley30056.pdf

Rohvein, C., Paravie, D., Urrutia, S., Roark, G., \& Nunes, D. (2013). Metodología de Evaluación 
del Nivel de Competitividad de las Pymes. Revista Ciencias Estratégicas.

Saavedra, M. (2012 ). Una Propuesta para la determinación de la competitividad en la pyme latinoamericana . Revista Pensamiento y Gestión No 33 .

Saavedra, M., \& Milla, S. (2012). La competitividad en el nivel micro de la PYME en el Estado de Queretaro. Revista En - Contexto No $7,176-203$.

Saavedra, M., Aguilar, M., \& Sanchez, B. (2015). La competitividad sistémica de la PYME del Distrito Federal, México . Revista FIR, FAEDPYME International Review No 4.

Saavedra, M., Milla, S. , \& Sanchez, B. (2013). Determinación de la competitividad de la PYME en el nivel micro: El caso del Distrito
Federal, México. Revista FAEDPYME International Review Vol 2 No 4, 38-52.

Solleiro, J., \& Castañon, R. (2005). Competitiveness and innovation systems: Dechallenges for Mexico's insertion in the global contex. Revista Technovation, 1059-1070.

Unidas-Cepal, N. (2001). Elementos de competitividad Sistémica de las Pequeñas y Medianas Empresas (PYME) del Istmo Centroamericano.

Usero, M., \& Fernandez, Z. . (2o de setiembre de 2018). Diferencias en la conducta competitiva de pioneros y seguidores. Análisis en el sector de la telefonía móvil en Europa. Investigaciones europeas de Dirección y Economía de Empresa, 2015. Obtenido de http://www.redalyc.org/articulo. oa ?id=274120419001 $>$ ISSN 